Transfusion Medicine
and Hemotherapy
Übersichtsarbeit - Review Article

Transfus Med Hemother 2008;35:431-437

DOI: $\underline{10.1159 / 000165781}$
Eingereicht: 21. Juli 2008

Angenommen: 30. Juli 2008

Published online: November 14, 2008

\title{
Augenhornhaut - Banken und klinische Anwendung
}

\author{
Jan Schroeter ${ }^{\mathrm{a}} \quad$ Peter Rieck $^{\mathrm{a}} \quad$ Philip Maier $^{\mathrm{b}} \quad$ Thomas Reinhard $^{\mathrm{b}}$ \\ ${ }^{a}$ Augenklinik, Charité - Universitätsmedizin Berlin, Campus Virchow-Klinikum, Berlin, \\ ${ }^{b}$ Augenklinik, Albert-Ludwigs-Universität Freiburg, Deutschland
}

\section{Schlüsselwörter \\ Hornhautbank · Hornhauttransplantation . \\ Gesetzliche Rahmenbedingungen}

\section{Zusammenfassung}

Das neue Gewebegesetz als Folge der Umsetzung der Richtlinien des EU-Parlaments und des Rates 2004/23/EG, 2006/17/EG und 2006/86/EG wird an den Prozessen in der Hornhautbank nichts grundlegend ändern. Es ist aber eine zunehmende Transparenz bei der Gewinnung und Prozessierung von Spenderhornhäuten, eine Harmonisierung bestimmter Arbeitsschritte und ein erhöhtes Bewusstsein für die Bedeutung validierter Abläufe zu erwarten. Bei der Umsetzung des Gesetzes müssen die Besonderheiten der humanen Augenhornhaut berücksichtigt werden, insbesondere ihre natürliche Unsterilität, fehlende Sterilisierbarkeit, postmortale Explantationszeit bis zu $72 \mathrm{~h}$ und ihre Transplantierbarkeit trotz Karzinomerkrankung und bakterieller Sepsis des Spenders. Der Tätigkeitsschwerpunkt der Hornhautbanken bleibt die Bereitstellung von Hornhauttransplantaten mit normaler physiologischer Funktionalität und minimalem Risiko einer Krankheitsübertragung. Die Steigerung der Anzahl transplantierbarer Spenderhornhäute ist zur Überwindung des weiterhin bestehenden Mangels dabei eine wesentliche Aufgabe.

\section{Entwicklung und aktueller Stand der Hornhauttrans- plantation in Deutschland}

Seit der ersten Beschreibung einer erfolgreichen Hornhauttransplantation durch Eduard Zirm 1906 [1] ist diese Therapie unverzichtbarer Bestandteil der Behandlung irreversibler Ver-

\author{
Key Words \\ Eye banks - Corneal transplantation . \\ Government regulation
}

\section{Summary}

Cornea - Banking and Clinical Application

The regulations in the recent German Tissue Act as a result of the implementation of the EC directives 2004/ 23/EC, 2006/17/EC and 2006/86/EC will not fundamentally change the processing in the eye bank, but will lead to an increasing transparency in procurement and processing of donor corneas, to a harmonization of certain procedures and an increasing awareness of the importance of validated techniques. The peculiarities of the human cornea, especially its non-sterility and impossibility of sterilization, death-to-explantation time of up to $72 \mathrm{~h}$ and transplantation despite of carcinoma and bacterial septicemia of the donor, have to be considered during the implementation of this act. The focus for the eye banks remains the supply of corneal transplants with normal physiological function and a minimal risk of disease transmission. An increase in the number of transplantable donor corneas is hereby an important challenge to overcome the present shortage.

änderungen der optischen Qualität, der Transparenz bzw. der strukturellen Integrität der Hornhaut geworden. Mikrochirurgische Weiterentwicklungen bis hin zum Einsatz der Femtosekundenlasertechnik [2], feines Nahtmaterial und moderne Trepansysteme haben den Erfolg der Keratoplastik im Laufe der Zeit deutlich verbessert (Abb. 1). Zusätzlich hat die Ein-

\section{KARGER}

Fax +497614520714

Information@Karger.de

www.karger.com
() 2008 S. Karger GmbH, Freiburg

Accessible online at:

www.karger.com/tmh

Dr. med. Jan Schroeter, FEBO

Augenklinik, Charité - Universitätsmedizin Berlin

Campus Virchow-Klinikum

Augustenburger Platz 1, 13353 Berlin, Deutschland

Tel. +49 30450 554-099, Fax -989

jan.schroeter@charite.de 
führung humaner Leukozytenantigen (HLA) gematchter Hornhauttransplantationen und der Einsatz immunmodulierender Medikamente die Prognose für bestimmte Patienten deutlich erhöht. Der gezielte Austausch erkrankter Hornhautstrukturen durch eine vordere bzw. hintere lamelläre Keratoplastik sowie die isolierte Descemetmembran/Endotheltransplantation versprechen eine weitere Verbesserung für die Versorgung der Patienten.

Vladimir Filatov war der Erste, der die Augenhornhäute Verstorbener zur Transplantation einsetzte. Er war es auch, der erstmals die entnommenen Spenderaugen in einer feuchten Kammer gekühlt für maximal 56 h bis zur Transplantation aufbewahrte und diesen Eingriff damit planbar machte. Diese Lagerungsmöglichkeit trug wesentlich zur Verbreitung der Keratoplastikoperation mit Hornhäuten von Verstorbenen bei. Im Laufe der Jahre wurden verschiedene Möglichkeiten der Lagerung isolierter Hornhaut-Skleralscheiben versucht, unter anderem die Aufbewahrung in Blutserum. Versuche mit verschiedenen Zellzuchtmedien führten 1974 zur Entwicklung eines Mediums durch McCarey und Kaufman [3], das weltweit die erste sichere Konservierung bei $+4{ }^{\circ} \mathrm{C}$ deutlich über $48 \mathrm{~h}$ hinaus ermöglichte. Von diesem Medium leiten sich letztlich alle heute gebräuchlichen Hornhautkonservierungsmedien für die hypotherme Kurzzeitkonservierung ab. Summerlin [4] berichtete 1973 erstmals über die Lagerung von Spenderhornhäuten unter physiologischen Bedingungen in der sogenannten Organkultur in einem Zellkulturmedium bei etwa $+32^{\circ} \mathrm{C}$. In Europa wurde diese Methode von Sperling [5] klinisch realisiert und fand mit der Etablierung der Hornhautbank in Amsterdam 1981 durch Pels und Schuchard [6] ihren Einsatz in die Routine. Das Organkulturverfahren ermöglicht eine Lagerung von Spenderhornhäuten bis zu 4 Wochen und damit den geplanten Einsatz in verschiedenen Kliniken und auch eine HLA gematchte Übertragung. Diese Konservierungsmethode setzte sich unter anderem deswegen in Europa durch und wird heutzutage von allen größeren Hornhautbanken eingesetzt. In Deutschland entstanden besonders Anfang der 1990er Jahre des letzten Jahrhunderts zahlreiche Hornhautbanken, die die Organkulturmethode einsetzen.

Während in den USA nach Gründung der ersten Augenbank durch R. Townley Paton in New York City 1944 relativ früh eigenständige Institutionen entstanden waren, die Spenderhornhäute für die Augenkliniken zur Verfügung stellten, entwickelten sich in Deutschland solche Banken erst deutlich später ab etwa 1975 als eigenständige Abteilungen an Universitätsaugenkliniken. Aktuell existieren in Deutschland 26 Hornhautbanken, die in einer Arbeitsgemeinschaft bei der Deutschen Ophthalmologischen Gesellschaft organisiert sind. Davon sind 20 an Universitätskliniken angesiedelt ( $w w w$. deutsche-hornhautbanken.de). Alle deutschen Hornhautbanken versorgen vorrangig die eigene Klinik, wobei etwa die Hälfte überzählige Hornhauttransplantate an andere Kliniken und Operateure abgibt. Unabhängig von den etablierten Hornhautbanken gewinnen einige Kliniken bzw. Operateure

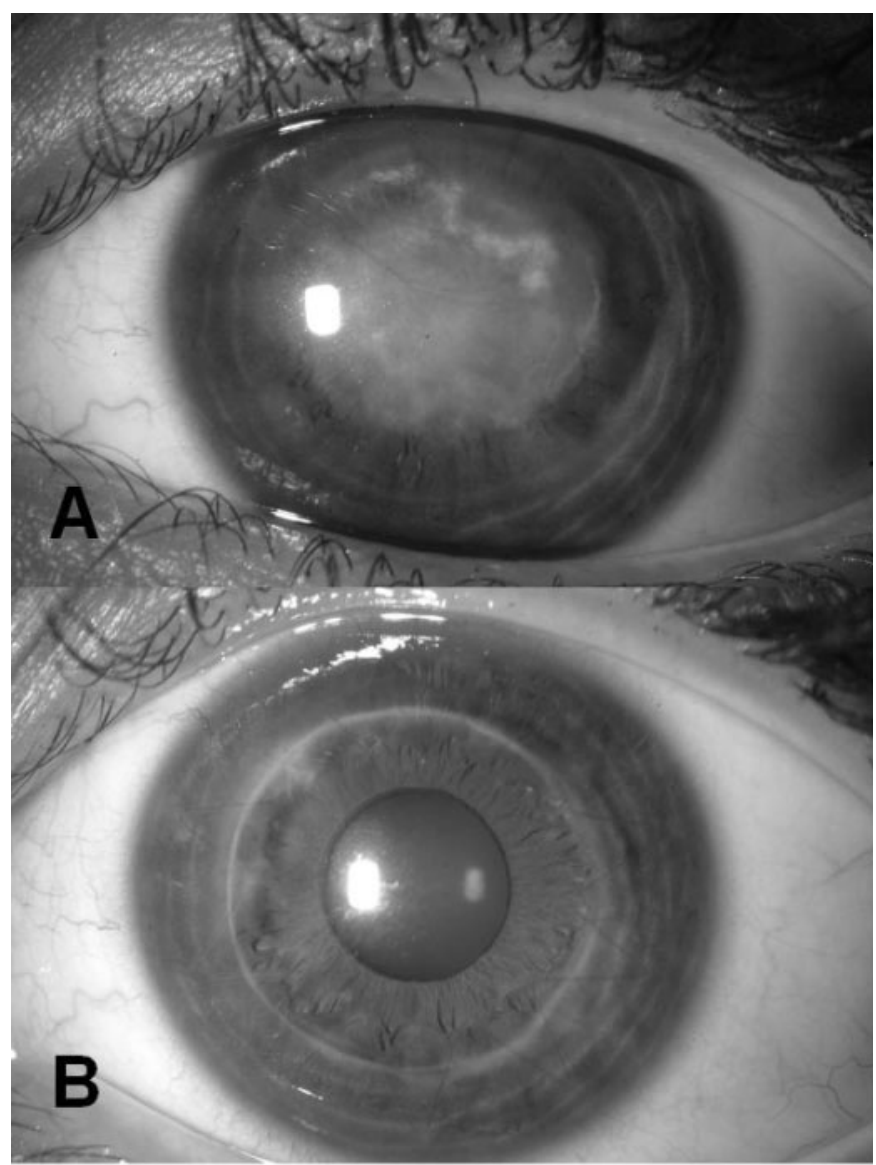

Abb. 1. Auge mit Hornhautnarbe durch Keratitis A vor und B nach penetrierender Keratoplastik mit doppelter Kreuzstichnaht nach Hoffmann.

selbstständig Spenderhornhäute, die dann entweder direkt oder nach Lagerung in einer feuchten Kammer transplantiert werden. Nach einer Umfrage unter deutschen Ophthalmochirurgen wurden 2003 etwa $22 \%$ der transplantierten Hornhäute so bereitgestellt [7].

In letzter Zeit werden jährlich etwa 5000 Keratoplastiken in der Deutschland durchgeführt.

Voraussetzung für die erfolgreiche Behandlung des Patienten ist die Bereitstellung eines geeigneten Hornhauttransplantates. Dies sollte eine normale physiologische Funktionalität besitzen und möglichst ohne Risiko einer Krankheitsübertragung sein. Darin besteht der Tätigkeitsschwerpunkt der Hornhautbanken.

\section{Gesetzliche Rahmenbedingungen}

Am 1. Dezember 1997 trat das erste deutsche Transplantationsgesetz (TPG) in Kraft, das die Abläufe der postmortalen Spende für alle Organe und viele Gewebe, wie auch die Augenhornhaut, grundsätzlich regelte. Die Spende und Entnahme der Augenhornhaut wurden danach vielerorts neu organisiert, was auch zu einer Verbesserung der Transplantatverfügbarkeit beitrug $[8,9]$. Die seither gültige erweiterte Zustim- 
mungslösung hat sich in der Praxis bewährt. Für die Transplantation der Augenhornhaut war dieses Gesetz von besonderer Bedeutung, so wurde sie doch explizit im $\S 2$ Abs. 3 Nr. 8 des damals novellierten Arzneimittelgesetzes (AMG) von der Anwendung des Arzneimittelrechts ausgenommen.

Nach Erlass des Transplantationsgesetzes erfolgte gemäß $§ 16$ des Gesetzes eine klare Strukturierung der Abläufe bezüglich der Entnahme und Organkultur humaner Augenhornhäute durch die entsprechenden Fachgesellschaften. Dies führte im Jahr 2000 zur Veröffentlichung der «Richtlinien zum Führen einer Hornhautbank» [10] durch die Bundesärztekammer und im Jahr 2001 zur Bekanntmachung der «Durchführungsbestimmungen der Arbeitsgemeinschaft Deutscher Hornhautbanken für die Kultivierung von Spender-Hornhäuten und die Organisation von Hornhautbanken» [11] durch die Deutsche Ophthalmologische Gesellschaft (DOG). Diese Dokumente besitzen bis heute Gültigkeit und enthalten bereits nahezu alle wichtigen Aspekte und Anforderungen des seit August 2007 geltenden Gewebegesetzes. Sie stellten auch die Grundlage für die Erstellung einer aktualisierten Richtlinie, der sogenannten «guten fachlichen Praxis (GFP)» (bisher noch unveröffentlicht), dar, die nach der Arzneimittel- und Wirkstoffherstellungsverordnung (AMWHV) für jede Gewebezubereitung formuliert werden muss. In dieser GFP werden die grundlegenden Standards für Hornhautbanken definiert, nach denen sich die Arbeit in allen deutschen Hornhautbanken orientieren muss und die die Grundlage für alle Begutachtungen einer Hornhautbank darstellen sollen.

Die Änderungen des Transplantationsgesetzes, die durch das seit dem 01. August 2007 in Kraft getretene «Gesetz über Qualität und Sicherheit von menschlichen Zellen und Geweben» (Gewebegesetz) vorgenommen wurden, werden grundsätzlich nur zu geringfügigen Änderungen der Prozesse in der Hornhautbank führen. So wurde in $\S 3$ Abs. 1 TPG eingeführt, dass die Entnahme von Geweben im Gegensatz zur Entnahme von Organen auch durch nicht ärztliches Personal unter ärztlicher Aufsicht durchgeführt werden darf.

Die in $\S 4$ klar definierte Auskunftspflicht wird erweitert, so dass nun auch einem Arzt, der eine postmortale Gewebespende realisieren möchte, in jeglicher Form Auskunft und Unterstützung gewährt werden muss.

Für Einrichtungen, in denen sowohl Organ- als auch Gewebespenden erfolgen oder mehrere Gewebe entnommen werden, ist § 7 TPG von Bedeutung, in dem klargestellt wird, dass die Einwilligung für alle Organe und alle Gewebe gleichzeitig und zusammen eingeholt werden muss. Für Organspenden bei Hirntoten fällt hier der Deutschen Stiftung Organtransplantation (DSO) als Koordinierungsstelle eine entscheidende Aufgabe zu. Wie in der Praxis routinemäßig verfahren werden soll, muss noch etabliert werden. Bei Herz-Kreislauf-toten Gewebespendern sollte die einzügige Einholung des Einverständnisses für die Entnahme verschiedener Gewebe durch die jeweils lokal und regional vorhandenen Gewebebanken nach vorhergehender Absprache erfolgen.
Weiterhin fordert $§ 8 \mathrm{~d}$ TPG neben einer ausführlichen Dokumentationspflicht mit Berichterstattung an das Paul-EhrlichInstitut einen angemessen ausgebildeten Arzt für jede Gewebeeinrichtung, der die Verantwortung für die Beurteilung des Spendereinschlusses und die Freigabe der Gewebe übernimmt. In § 9 TPG wird der Vorrang von Organen vor Geweben definiert, so dass Augenhornhäute erst dann entnommen werden dürfen, wenn die Organentnahme nicht möglich oder durch die Gewebeentnahme nicht beeinträchtigt wird. Schließlich bleibt es durch $\S 17$ bei einem strikten Handelsverbot auch für Gewebezubereitungen, die als Arzneimittel definiert sind.

Die für Hornhautbanken entscheidende Änderung durch das Gewebegesetz ist die Abwandlung von $\S 2$ Abs. 2 Nr. 8 AMG, wonach die ehemals explizit den Organen zugerechnete $\mathrm{Au}-$ genhornhaut ihren Organstatus verliert und schließlich in $\S 4$ Abs. 30 AMG als Gewebezubereitung, also als ein Arzneimittel, definiert wird. Hierdurch bestand die erste Hürde für die etablierten Hornhautbanken in der Beantragung einer Gewinnungs- und Herstellungserlaubnis gemäß $\S 20$ b und $20 \mathrm{c}$ AMG für Hornhauttransplantate bei der jeweils lokal zuständigen Behörde. Die Voraussetzungen für die Erteilung einer solchen Erlaubnis wurden vom Gesetzgeber im Gesetzestext grob skizziert. Die konkrete Umsetzung bezüglich der Anforderungen an eine Hornhautbank - dies lässt sich bereits jetzt sagen - wird unter den verschiedenen Bundesländern jedoch voraussichtlich deutlich variieren, da die Gesetzesvorgaben einen gewissen Interpretationsspielraum zulassen. Hier wäre ein einheitliches Vorgehen vor allem mit Berücksichtigung der unten beschriebenen Besonderheiten der Augenhornhaut wünschenswert. Neben jeder einzelnen Hornhautbank müssen auch alle Labore oder Einrichtungen, die Teilleistungen für die Bereitstellung der Hornhauttransplantate erbringen, einen Antrag nach $\$ 20$ b AMG stellen oder einen Vertrag mit der jeweiligen Hornhautbank abschließen. Hierzu zählen zum Beispiel Labore, die die infektiologischen Untersuchungen durchführen. Neben diesen beiden Anträgen bei den lokalen Behörden wurde für die Augenhornhaut als klassische Gewebezubereitung in $\S 21$ a AMG ein vereinfachtes Zulassungsverfahren beim Paul-Ehrlich-Institut eingeführt. Ob allerdings tatsächlich jede Hornhautbank einer solchen Genehmigung bedarf, geht aus dem Gesetzestext nicht klar hervor. Denn es ist nicht eindeutig geklärt, wie der Begriff «Inverkehrbringen» definiert ist, da dieser sowohl in $\S 20 \mathrm{c} \mathrm{AMG}$ als auch in $\S 21 \mathrm{a}$ AMG genannt wird. Hierbei wurden bislang verschiedene Interpretationen und Auffassungen bezüglich dieses Begriffs diskutiert, so dass es auch denkbar erscheint, dass nach Erhalt einer Herstellungserlaubnis nach § 20 c AMG durch die lokale Behörde eine Verwendung der Gewebe für Transplantationszwecke innerhalb der eigenen Abteilung auch ohne Genehmigung nach $\S 21$ a AMG möglich ist. Die Entscheidung hierüber scheint derzeit bei den lokal zuständigen Behörden zu liegen, die die Herstellungserlaubnis nach § 20 c AMG im Benehmen mit dem Paul-Ehrlich-Institut erteilen. Hier bleibt zu hoffen, dass in naher Zukunft eine grundsätzliche juristi- 
sche Klärung dieses Sachverhaltes durch den Gesetzgeber erfolgt. Um das Antragsverfahren sowohl für das Paul-EhrlichInstitut als auch für die zahlreichen deutschen Hornhautbanken zu vereinfachen, wurde von der Arbeitsgemeinschaft Deutscher Hornhautbanken der DOG beschlossen, unter der Federführung der Charité Berlin einen gemeinsamen Musterantrag zu erarbeiten. Dieser soll mit professioneller Hilfe in das passende modulare Format gebracht werden, so dass jede Hornhautbank nur noch kleinere Anpassungen vornehmen muss. Dies ist nicht nur mit einem erheblichen personellen Aufwand für die einzelnen Hornhautbanken verbunden, sondern auch mit finanziellen Belastungen, die sowohl durch die Inanspruchnahme von professioneller Hilfe bei der Erarbeitung des Antrags als auch durch die Antragsbearbeitung beim Paul-Ehrlich-Institut entstehen. Wie diese finanziellen Belastungen ausgeglichen werden sollen, lässt der Gesetzgeber offen, der zwar bei der Verabschiedung des Gewebegesetzes keine finanzielle Belastung der öffentlichen Haushalte sieht, die Belastungen der einzelnen Einrichtungen jedoch unberücksichtigt lässt. Auch hier müssen in Zukunft die bisher nicht dargestellten oder neu entstehenden Kosten über eine Anpassung der diagnosebezogenen Fallgruppen (DRG) durch das Institut für das Entgeltsystem im Krankenhaus (INEK) auf die Allgemeinheit verteilt werden.

Die AMWHV findet mit der Definition der Augenhornhaut als Gewebezubereitung grundsätzlich Anwendung auf alle Abläufe in einer Hornhautbank. In einer Änderung der AMWHV vom 26. März 2008 wurden spezifische Regelungen für Gewebeeinrichtungen berücksichtigt, die einer Erlaubnis nach den neu eingeführten $\$ \S 20$ b und 20 c AMG bedürfen.

\section{Besonderheiten der Augenhornhaut bei Entnahme und Prozessierung}

Für die Spende einer Augenhornhaut kommt, abgesehen von den anerkannten Kontraindikationen, nahezu jeder Verstorbene unabhängig von dessen Alter auch noch bis zu $72 \mathrm{~h}$ nach dem Tode infrage. Insbesondere stellen Karzinomerkrankungen im Gegensatz zu allen anderen postmortalen Gewebespenden keine Kontraindikation für die Augenhornhaut dar, da eine Übertragung auf den Empfänger bei fehlender Augenbeteiligung ausgeschlossen ist.

Bei der Augenhornhaut handelt es sich grundsätzlich nicht um keimfreies Gewebe. Es finden sich vielmehr auf der Bindehaut und auf der Hornhautoberfläche bei allen Menschen zahlreiche mikrobielle, zum größten Teil jedoch apathogene Erreger. Die Epithelseite der Augenhornhaut kann zwar mit geeigneten Substanzen wie zum Beispiel Polyvinylpyrrolidon (PVP)-Jod desinfiziert, aber weder bei der Entnahme noch zu irgendeinem späteren Zeitpunkt während der Konservierung sterilisiert werden, ohne einen kompletten Funktionsverlust zu verursachen. Es kommen daher stets Keime mit dem Transplantat in das Organkulturmedium, deren Anzahl durch Ver- dünnung und den Zusatz von Antibiotika und Antimykotika jedoch stark reduziert und somit in der Regel unter die Keimnachweisgrenze gesenkt wird. Um dieser grundsätzlichen Keimbelastung der Augenhornhaut gerecht zu werden, zeigen regelmäßige mikrobiologische Kontrollen der Nährmedien während der Organkultur eine signifikante und somit für den Patienten potenziell gefährliche Keimlast an, so dass in diesen Fällen die betroffenen Augenhornhäute nicht für die Transplantation freigegeben werden. Diese hohe Sicherheit vor mikrobiologischen Infektionen der Spenderhornhaut macht die Organkultivierungsmethode (Aufbewahrung der Spenderhornhaut in einem Brutschrank bei etwa $34{ }^{\circ} \mathrm{C}$ ) im Vergleich zur hypothermen Konservierung (Aufbewahrung der Spenderhornhaut im Kühlschrank bei etwa $4{ }^{\circ} \mathrm{C}$ ), bei der keine Sterilitätstestungen vorgenommen werden, überlegen. Aus diesem Grund kommen gemäß der EU Direktive 2006/17/EC, Anhang 1, Punkt 1.1.5 im Gegensatz zu den meisten anderen Organen und Geweben auch an einer bakteriellen Sepsis Verstorbene für eine Hornhautspende explizit in Frage, sofern eine Organkultivierung des Spendergewebes erfolgt [12]. Weiterhin ist die Augenhornhaut im Gegensatz zu den meisten anderen Geweben nach der Transplantation nach außen hin stets sichtbar und kann so hinsichtlich unerwünschter Ereignisse wie zum Beispiel Infektionen oder Abstoßungsreaktionen auch nach der Verpflanzung ohne größere Belastung für den Patienten durch einen Augenarzt kontrolliert werden. Obwohl in verschiedenen Studien gezeigt werden konnte, dass bei der Transplantation nicht selten Keime mit übertragen werden [13-16], ist die Häufigkeit einer Endophthalmitis, also einer bakteriellen oder mykotischen Infektion des Augeninneren, nach Hornhautverpflanzungen sehr gering. In einer Umfrage unter den Mitgliedern der Arbeitsgemeinschaft Deutscher Hornhautbanken der DOG ergab sich eine Endophthalmitisrate bei organkultivierten Hornhauttransplantaten von 0,035\% (bisher unveröffentlichte Daten). In der Literatur werden Größenordnungen um $0,38 \%$ angegeben, allerdings auch unter Einbeziehung von Daten zu hypotherm konservierten Hornhauttransplantaten. Auch ist die Rate an postoperativen Infektionen nicht größer als die nach anderen intraokularen Eingriffen [17].

Aus den beschriebenen Gründen sind die immer wieder für die Herstellung von Geweben geforderten Reinräume für die Organkultur von Augenhornhäuten nicht sinnvoll, sondern würden nur zu einer von den wenigsten Hornhautbanken zu bewältigenden finanziellen Belastung führen, ohne eine höhere Sicherheit für die Patienten zu bringen. Dieser Besonderheit der Augenhornhaut wird der Gesetzgeber gemäß der EU Direktive 2006/86/EC (Anhang 1, Teil D, Punkt 4) in $§ 36$ Abs. 2 Nr. 2 der AMWHV gerecht, indem er hier bewusst Entscheidungsspielraum lässt, so dass unter bestimmten Vorraussetzungen auch undefinierte Hintergrundsluft in Gewebebanken zulässig ist.

Da es sich beim größten Teil der Hornhautspender um HerzKreislauf-Tote und nicht wie bei Organspendern um Hirntote 


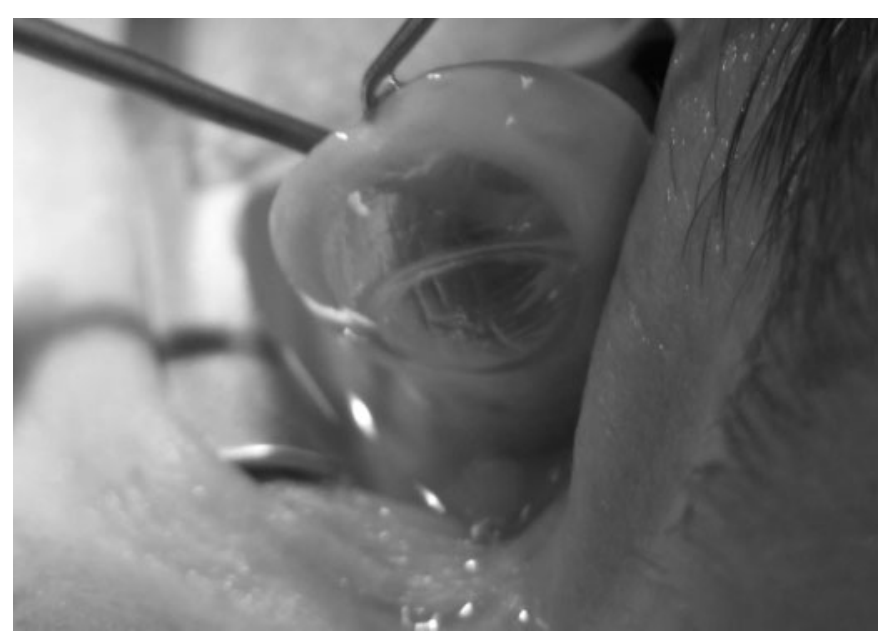

Abb. 2. Entnahme des isolierten Corneo-Sklera-Komplexes bei einem Hornhautspender.

handelt, ist ein Operationssaal als Entnahmeort nicht realisierbar. Dies ist auch aufgrund der oben beschriebenen stets vorhandenen bakteriellen Besiedlung von Horn- und Bindehaut nicht notwendig, da die Entnahme sterilen Gewebes grundsätzlich unmöglich ist. Die Entnahme der Spenderhornhaut bzw. des -auges erfolgt analog zu einem ophthalmochirurgischen Eingriff an einem lebenden Patienten nach ordnungsgemäßer chirurgischer Händedesinfektion und Anlegen steriler Kleidung sowie steriler Handschuhe, Gesichtsmaske und Kopfschutz unter Verwendung steriler Instrumente in einem lokalen sterilen Bereich durch Abdecken mit sterilen Tüchern, nachdem eine wirksame Haut- und Bindehautdesinfektion mit einem geeigneten Desinfektionsmittel (z.B. PVPJod) erfolgt ist (Abb. 2).

Unter diesen Voraussetzungen kann die Hornhautentnahme an nahezu allen Orten, die hygienischen Mindestanforderungen gerecht werden, so zum Beispiel in Räumlichkeiten der Institute für Pathologie oder Rechtsmedizin, stattfinden. Um einen Rückgang der Hornhautentnahmen zu vermeiden, muss es auch weiterhin möglich sein, an diesen Orten Hornhautentnahmen durchführen zu können. Dieser Möglichkeit einer Flexibilität hinsichtlich der Entnahmeorte wird in § 34 Abs. 2 der AMWHV gemäß der EU Direktive EC 200617EC, Anhang 4, Punkt 1,3 Rechnung getragen.

Die Entnahme einer vitalen Augenhornhaut ist auch noch bis zu 72 h nach einem Herz-Kreislauf-Stillstand möglich, da die Augenhornhaut nicht vaskularisiert und damit auch nicht in dem Maße wie die meisten anderen Organe und Gewebe von der Blutzirkulation abhängig ist. Demgegenüber gibt die EUDirektive 2006/17/EC, Anhang 2, Punkt 2.4 vor, dass Blutproben zur Testung auf übertragbare Erkrankungen von verstorbenen Gewebespendern nicht später als $24 \mathrm{~h}$ nach dem Tode gewonnen werden dürfen, so dass hier ein neues Ausschlusskriterium geschaffen wurde, sofern nicht auf eine prämortale Blutprobe des Spenders zurückgegriffen werden kann. Dies

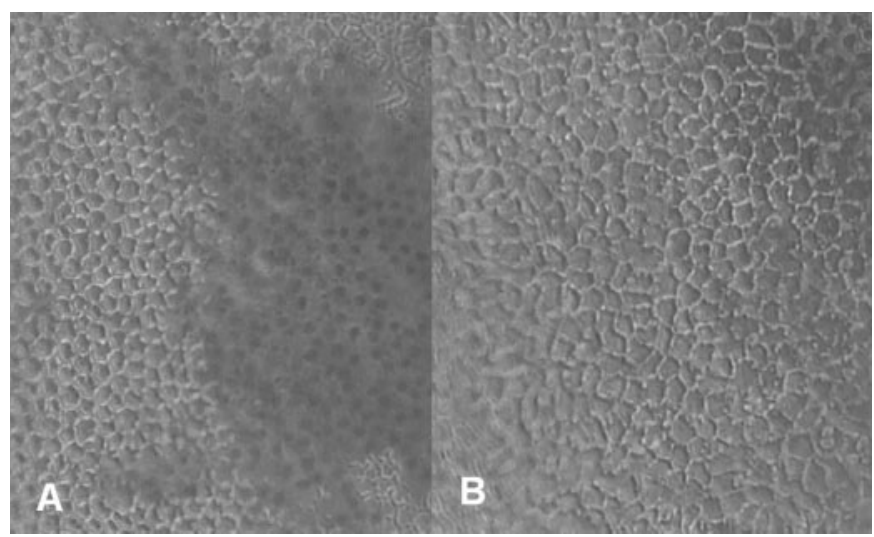

Abb. 3. Endothelzellschicht einer Spenderhornhaut A vor und B nach 18-tägiger Organkultivierung. A Rechts im Bild sind deutlich großflächige Endothelzellnekrosen zu erkennen. B Die Endothelzellschicht zeigt nach Wundheilungsvorgängen eine reduzierte Zelldichte und Veränderung der Zellmorphologie.

erscheint in Anbetracht der 72-Stunden-Grenze für Hornhautentnahmen überdenkenswert, da so zukünftig zahlreiche, potenziell geeignete Hornhautspender nicht mehr für eine Hornhautentnahme infrage kommen.

Die Avaskularität der Hornhaut macht auch die Übertragung einer viralen Hepatitis bzw. einer HIV-Infektion sehr unwahrscheinlich. Seit der Einführung der routinemäßigen serologischen Testung für Hornhautspender wurden keine Übertragungen von HBV berichtet und für HCV und HIV gibt es in der gesamten Literatur bis heute keinen einzigen Bericht über eine Übertragung durch eine Hornhauttransplantation, obwohl vereinzelt Hornhäute von HIV-positiven Spendern übertragen worden waren [18-20]. Weiterhin konnte im Organkulturmedium und im Hornhautgewebe von serologisch HBVund HCV-positiven Spendern in weniger als der Hälfte der Fälle Virus-DNA in sehr geringen Mengen nachgewiesen werden, so dass eine potenzielle Übertragung durch eine Hornhauttransplantation selbst bei positiven serologischen Tests fraglich erscheint [21]. In der Richtlinie der Bundesärztekammer zum Führen einer Hornhautbank wird die HCV-RNATestung im Serum mittels PCR gefordert. Damit ist Deutschland das einzige europäische Land, in dem diese Untersuchung bei Hornhautspendern gefordert ist. Die EU Direktive 2006/17/EC, die deutlich später in Kraft getreten ist, fordert diesen Test nicht. Hinsichtlich der sehr fraglich erhöhten Sicherheit für die Empfänger von Hornhauttransplantaten und des nicht unerheblichen Verlustes von Spendergewebe durch falsch-positive bzw. unklare Testergebnisse durch Veränderungen im postmortalen Spenderserum sollte die Forderung nach der HCV-RNA-Testung in Deutschland kritisch überprüft werden.

Die Organkulturmethode erlaubt regenerative Prozesse des Gewebes, so dass Spenderhornhäute durch die Konservierung nahezu ihren physiologischen Zustand wiedererlangen können, aber auch irreversible Schädigungen, die gegen eine 
Transplantation sprechen, erkennbar werden. Damit bietet diese Konservierungsmethode einen weiteren Sicherheitsaspekt (Abb. 3).

Die Spenderhornhaut wird am Ende der Kultivierungsperiode vor Freigabe zur Transplantation einer kritischen Evaluation unterzogen. Neben einer unauffälligen Spenderanamnese ohne Anhalt für Kontraindikationen, negativer Infektionsserologie und unauffälligen Sterilitätsproben des Kultivierungsmediums wird eine biomikroskopische und lichtmikroskopische Prüfung vorgenommen. Damit werden morphologische Veränderungen wie Narben, z.B. als Folge von Verletzungen, und Trübungen des Hornhautstromas erkannt. Lichtmikroskopisch werden mithilfe einer osmotischen Stimulation die Endothelzellen visualisiert und ihre Dichte bestimmt. Nur Spenderhornhäute mit einer physiologisch ausreichenden Endothelzelldichte von mindestens 2000 Zellen $/ \mathrm{mm}^{2}$ und morphologisch unauffälliger Endothelzellschicht (z.B. kein Nachweis sogenannter Guttae) werden für eine elektive Transplantation freigegeben. Damit bietet diese Qualitätskontrolle am Ende der Konservierungsperiode einen weiteren, wesentlichen Sicherheitsaspekt für den Empfänger eines Hornhauttransplantates.

\section{Finanzierung}

Die Kosten für ein Hornhauttransplantat werden in Deutschland in den 2003 eingeführten Fallpauschalen (DRG) nicht korrekt abgebildet. So werden diese im Jahr 2008 mit zirka 863,- EUR beziffert. Tatsächlich zeigen neueste Schätzungen jedoch, dass die Kosten, die für die Bereitstellung eines Hornhauttransplantat von der Entnahme bis zur Transplantation entstehen, bei etwa 1700,- EUR liegen. Wie diese Differenz ausgeglichen werden soll, bleibt bisher jeder Hornhautbank selbst überlassen, so dass die meisten Hornhautbanken auf die finanzielle Unterstützung durch andere Erlöse der Augenkliniken oder Drittmittel bzw. Spenden angewiesen sind. Die oben genannten Schätzungen beziehen allerdings noch nicht die zusätzlichen Kosten für ein Hornhauttransplantat mit ein, die durch die Umsetzung des Gewebegesetzes entstehen werden. Wie hoch diese tatsächlich sein werden, lässt sich bisher noch nicht abschätzen. In Zukunft müssen alle Kosten, die bisher nicht ausreichend abgedeckt werden, über eine Anpassung der DRG korrekt abgebildet und so auf die gesamte Gesellschaft verteilt werden.

\section{Ausblick}

Sämtliche Auswirkungen des Gewebegesetzes auf die Entnahme und Transplantation von Augenhornhäuten lassen sich zum jetzigen Zeitpunkt noch nicht endgültig abschätzen. Die zunehmende Transparenz in der Gewinnung und Prozessierung von Spenderhornhäuten, die zu erwartende Harmonisierung bestimmter Arbeitsschritte und ein erhöhtes Bewusstsein für die Bedeutung validierter Abläufe sind sicher von Vorteil. Es ist schwer vorstellbar, dass die damit verbundene Bürokratisierung und die aufwendigen Antragsverfahren in einem guten Verhältnis zum Nutzen, insbesondere für den Empfänger von Hornhauttransplantaten, stehen.

Es bleibt zu hoffen, dass die Besonderheiten der Augenhornhaut im Vergleich zu den meisten anderen Geweben bei den Antragsverfahren in den unterschiedlichen Behörden berücksichtigt werden. Hierbei sollte der vom Gesetzgeber eingeräumte Entscheidungsspielraum (z.B. für die Notwendigkeit von Reinräumen oder die Flexibilität von Entnahmeorten) voll ausgeschöpft werden, um einen Rückgang der Hornhautspenden und so eine Verlängerung der Wartezeiten auf ein Hornhauttransplantat für die Patienten zu vermeiden. Die zu erwartenden deutlich steigenden Kosten für die Hornhautbanken müssen in der Zukunft noch besser in der durch das INEK berechneten DRG abgebildet werden, um die finanzielle Belastung nicht ausschließlich auf die Hornhautbanken, sondern auf die gesamte Gesellschaft zu verteilen.

\section{Literatur}

1 Zirm, E: Eine erfolgreiche totale Keratoplastik. Albrecht Von Graefes Arch Klin Exp Ophthalmol 1906;64:580-593.

2 Meltendorf C, Schroeter J, Bug R, Kohnen T, Deller $\mathrm{T}$ : Corneal trephination with the femtosecond laser. Cornea 2006;25:1090-1092.

3 McCarey BT, Kaufman HE: Improved corneal storage. Invest Ophthalmol Vis Sci 1974;13:165-173.

4 Summerlin WT, Miller GE, Harris JE, Good RA: The organ-cultured cornea. An in vitro study. Invest Ophthalmol Vis Sci 1973;12:176-180.

$\checkmark 5$ Sperling S: Human corneal endothelium in organ culture. The influence of temperature and medium of incubation. Acta Ophthalmol (Copenh) 1979;57: 269-276.

6 Pels E, Schuchard Y: Organ culture preservation of human corneas. Doc Ophthalmol 1983;56:147-153.
7 Bertelman E, Reinhard T, Pleyer U: Current practice of immune prophylaxis and therapy in perforating keratoplasty. A survey of members of the Cornea Section of the German Ophthalmological Society. Ophthalmologe 2003;100:1031-1035.

8 Reinhard T, Sundmacher R: Hornhauttransplantationen: Überwindung des Engpasses. Dtsch Ärztebl 2000;97:1452-1455.

9 Reinhard T, Bohringer D, Bogen A, Sundmacher R: The transplantation law: a chance to overcome the shortage of corneal grafts in Germany? Transplant Proc 2002;34:1322-1324.

10 Bachmann KD, Eisenmenger W, Engelmann K, Gerlich WH, Persijn G, Püschel K, Schaal K, Sewing KF, Sundmacher R, Winter R, Witschel H, Wollersheim U, Winter S: Richtlinien zum Führen einer Hornhautbank. Dtsch Ärztebl 2000;97:21222124.
11 Durchführungsbestimmungen der Arbeitsgemeinschaft Deutscher Hornhautbanken für die Kultivierung von Spender-Hornhäuten und die Organisation von Hornhautbanken 2001, www.dog.org/ publikationen/hornhautbanking.html.

12 Spelsberg H, Reinhard T, Sengler U, Daeubener W, Sundmacher R: Organ-cultured corneal grafts from septic donors: a retrospective study. Eye 2002;16: 622-627.

13 Albon J, Armstrong M, Tullo AB: Bacterial contamination of human organ-cultured corneas. Cornea 2001;20:260-263.

14 Farrell PL, Fan JT, Smith RE, Trousdale MD: Donor cornea bacterial contamination. Cornea 1991;10:381-386.

15 Ritter E, Gotze J, Trute K, Strache S, Schmidt G, Gliem H: The extent of bacterial contamination of keratoplasty donor eyes post mortem. Klin Monatsbl Augenheilkd 1990;196:70-75. 
16 Gomes JA, Dana MR, Dua HS, Goren MB, Laibson PR, Cohen EJ: Positive donor rim culture in penetrating keratoplasty. Cornea 1995;14:457-462.

17 Taban M, Behrens A, Newcomb RL, Nobe MY, McDonnell PJ: Incidence of acute endophthalmitis following penetrating keratoplasty: a systematic review. Arch Ophthalmol 2005;123:605-609.

18 Hoft RH, Pflugfelder SC, Forster RK, Ullman S, Polack FM, Schiff ER: Clinical evidence for hepatitis B transmission resulting from corneal transplantation. Cornea 1997;16:132-137.

19 Glasser DB: Serologic testing of cornea donors. Cornea 1998;17:123-128.

20 Simonds RJ, Holmberg SD, Hurwitz RL, et al: Transmission of human immunodeficiency virus type 1 from a seronegative organ and tissue donor. N Engl J Med 1992;326:726-732.

21 Sengler U, Reinhard T, Adams O, Gerlich W, Sundmacher R: Testing of corneoscleral discs and their culture media of seropositive donors for hepatitis $\mathrm{B}$ and $\mathrm{C}$ virus genomes. Graefes Arch Clin Exp Ophthalmol 2001;239:783-787. 